\title{
Goodness of regularity in dot patterns: global symmetry, local symmetry, and their interactions
}

\author{
Massimo Nucci \\ Dipartimento di Psicologia Generale, Università di Padova, via Venezia 8, I 35100 Padua, Italy; \\ e-mail: massimo.nucci@unipd.it \\ Johan Wagemans \\ Laboratory of Experimental Psychology, University of Leuven, Tiensestraat 102, B 3000 Leuven, \\ Belgium; e-mail: johan.wagemans@psy.kuleuven.be \\ Received 22 March 2005, in revised form 13 February 2007; published online 10 September 2007
}

\begin{abstract}
Goodness is a classic Gestalt notion defined as salience or perceptual strength of a given pattern. All operational models of goodness have assigned a central role to mirror symmetry but not much attention has been paid to the distinction between global and local mirror symmetry, and their possible interactions. We designed eight different types of dot patterns (all consisting of 80 dots), combining different numbers $(0,1$, and 2) and relative orientations (parallel or orthogonal to each other) of local and global axes of symmetry (affecting $50 \%$ or $100 \%$ of the dots, respectively) at different absolute orientations (vertical and horizontal). Each of 640 trials consisted of a short presentation of a new dot pattern, which subjects had to classify as regular or random. We hypothesised that the overall goodness of patterns is not the simple sum of the amount of regularity present in them but depends on the cooperation and competition between symmetries. The results confirmed our hypothesis, showing that performance in this regularity-detection task did not increase in a linear way when some symmetries were added to other symmetries.
\end{abstract}

\section{Introduction}

The Gestalt concept of goodness has strong intuitive roots closely related to the notion of 'Prägnanz' (Koffka 1935). The first Gestalt psychologists defined goodness as a perceptual attribute related to the perceptual strength or salience of a given pattern. More recently, this notion has changed somewhat and the goodness of a pattern is now defined in more operational terms as "the detectability of a global regularity and the effect thereon of noise and local regularities" (van der Helm and Leeuwenberg 2004, page 266).

One of the classical issues in goodness of regularity concerns the large distinction between mirror symmetry (henceforth symmetry) and repetition (Palmer 1982, 1983; Wagemans et al 1993; van der Helm and Leeuwenberg 1991, 1996). Indeed, many researchers have shown that detection of symmetry is faster and more accurate than detection of a simple repeated pattern (Mach 1886; Julesz 1971; Corballis and Roldan 1974; Bruce and Morgan 1975; Royer 1981; Foster and Kahn 1985; for a review, see Wagemans 1995). But another factor which might affect the discriminability of patterns is whether the perceived elements, which give rise to the regularity, belong to the same object or not. $^{(1)}$ In fact, it has been shown (Baylis and Driver 1995; Bertamini et al 1997) that symmetry is easier to detect within an object (therefore it is called a 'within-object relation'), while repetition is easier to detect when the matching parts belong to different objects (therefore it is called a 'between-object relation'). It is important to notice that this factor is so strong that it may actually reverse the classical advantage in goodness from symmetry to repetition (see also Koning and Wagemans 2008).

(1) We use the term 'pattern' to denote the overall figure or stimulus and the term 'object' for each visually distinct unit in the pattern. 
The issue of 'within- versus between-object relations' shows how the interaction between objectness and regularities plays a heavy role in the overall goodness of patterns. Hence, it appears natural to suppose that also the interaction between regularities themselves has an actual impact on the goodness of patterns. The presence of several symmetries could lead to different configurations of the overall figure because it is known that symmetry tends to constitute a distinct visual object (Locher and Wagemans 1993; Field et al 2000). Similarly, the number and the orientation of the axes of symmetry affect the structure and then the goodness of the figure. In a more general way, the overall configuration of a pattern, and consequently its goodness, depends on the interaction of its component parts and not just on the individual elements themselves (as Gestalt psychology has pointed out, of course). The aim of this paper is thus to take into account the interaction between symmetries in the overall goodness of regularity of dot patterns.

\subsection{Holographic approach}

In line with the more recent ideas about goodness, models have been proposed to describe (and predict) the goodness of regularity in a broad set of figures (Palmer 1982, 1983; van der Helm and Leeuwenberg 1991, 1996; Wagemans et al 1993). One of the most simple and powerful ways to quantify goodness is the holographic approach (van der Helm and Leeuwenberg 1991, 1996). Several researchers have investigated if this model really constitutes an accurate method to predict goodness; others have used it more as a heuristic basis for new research (Wenderoth and Welsh 1998; van der Helm and Leeuwenberg 1999, 2004; Wagemans 1999; Csatho et al 2003, 2004; Dastani and Scha 2003; Olivers et al 2004). In the present study we used the assumptions and the predictions of the holographic approach (henceforth HA), so let us summarise them briefly.

According to the HA, perceptual goodness of a regularity is based on the 'weight of evidence' $(W)$ for it. About goodness and 'weight of evidence', van der Helm and Leeuwenberg (1996, page 444) proposed the following definitions:

"The goodness of a pattern is, in our view, determined by the 'strength' of the regularity described in the simplest description of the pattern. By strength, we mean the amount of support, or 'weight of evidence', for the existence, as given by the identities that constitute this regularity. Because regularity is always embedded in a pattern, we propose to quantify goodness by $W=E / M$, in which $E$ is the number of holographic identities that constitute the regularity, whereas $M$ is the total information in the pattern."

The value of $W$ thus requires the computation of the number of holographic identities between local elements, $E$, and the computation of the total amount of information, $M$. The procedure to quantify $E$ and $M$ follows precise rules and becomes as complex as the pattern structure is. The former value, $E$, depends on a mathematical formalisation of the regularities embedded in a pattern (van der Helm and Leeuwenberg 1996, 2004), the latter value, $M$, is provided by structural information theory (henceforth SIT-Leeuwenberg 1969, 1971). Skipping the greater part of these procedures, let us focus on the calculation of the $W$ value for symmetric dot patterns (for a full characterisation of all the procedures of HA, see van der Helm and Leeuwenberg 1996; for a shorter version, see Wagemans 1999). With this kind of patterns, the number of holographic identities, $E$, is simply half of the number of dots implied in the symmetry, whereas the total amount of information, $M$, is equal to the number of the dots in the pattern. For instance, in a pattern with a simple global symmetry, that is with $100 \%$ of the dots implied in the symmetry, the $W$ value is $W=E / M=(n / 2) / n=\frac{1}{2}$, while in patterns which show $50 \%$ of dots implied in the symmetry, the $W$ value is $W=E / M=\left(\frac{1}{2} n / 2\right) / n=(n / 4) / n=\frac{1}{4}$.

For patterns that show multiple regularities, the HA proposes the same calculations, although the number of holographic identities, $E$, depends on the mathematical 
formalisation provided by SIT and varies as a function of the kind of regularity and their disposition inside the pattern (see van der Helm and Leeuwenberg 1996). For simpler configurations, van der Helm and Leeuwenberg (1996, page 446) affirm: "We simply assume that the $W$ values of compatible regularities are additive, as there is no reason to assume something more complex". The 'compatible regularities' are the ones captured in the mathematical formalisation provided by SIT. From an intuitive point of view, symmetries could be called compatible when they do not partially overlap. For instance, both the left half and the right half of figure $2 \mathrm{a}$ have a local symmetry and the two pattern halves do not overlap. The same is true for the two pattern halves in figure $2 \mathrm{c}$ but these two local symmetries are themselves nested within an overall symmetry that applies to the whole pattern, and that will have an implication for its overall goodness. All the multiple symmetries used in this study can be regarded as compatible regularities according to this view.

Let us consider some simple examples of the calculation of $W$ for those patterns. The $W$ value of a pattern with two disjoint local symmetries is simply the sum of the $W$ values of one and the $W$ value of the other. For instance, a double local symmetry where each one implies $50 \%$ of the dots (like in the pattern shown in figure $2 \mathrm{a}$ ) has $W=E / M=\frac{1}{4}+\frac{1}{4}=\frac{1}{2}$. The $W$ value of a pattern with two nested symmetries is the sum of the $W$ value due to one symmetry and the $W$ value of the other one, calculated as a local symmetry of a half-pattern. For instance, the $W$ value of the pattern shown in figure 2c is $W=\frac{3}{4}$, because of the sum of $W=\frac{1}{2}$, due to one global symmetry, and $W=\frac{1}{4}$, due to the remaining nested symmetry considered just one time as a local symmetry of a half-pattern. In table 1 one can find all the $W$ values of the patterns used in our experiment. It is good to remember that a higher value of $W$ implies a higher perceived goodness.

\subsection{Local and global symmetry and their interactions}

Local mirror symmetry can be defined as symmetry that does not affect the whole figure. The four dot patterns in figures $1 \mathrm{a}-1 \mathrm{~d}$ differ from one another by the percentage of dots implied in the symmetry $(25 \%, 50 \%, 75 \%$, and $100 \%$, respectively). It is self-evident that the lower the number of dots implied in the symmetry, the lower the perceived goodness of the whole pattern. The HA captures these differences nicely: it explains the increase of goodness as a consequence of the higher amount of regularity (holographic identities) in comparison with the overall amount of information.

Consider a pattern with two local symmetries with parallel axes, as shown in figure $2 \mathrm{a}$. In this kind of pattern, the number of dots implied in the symmetries is equal to the one involved in a simple global symmetry (like in figure 1d). Actually, in both patterns $100 \%$ of the dots are included in the regularities. In this case, the HA yields the same weight of evidence $W$ for both patterns and it thus predicts that they have the same goodness. One might argue, however, that other factors come into play that may complicate a direct comparison (see Wagemans 1995). For example, it is known that the distance between dots and their axis of symmetry affects the detectability of symmetry (Corballis and Roldan 1974; Foster and Kahn 1985; Wenderoth 1995). More precisely, the goodness increases as a function of proximity between dots and their symmetry axis. In figure $2 \mathrm{a}$ more dots are closer to their local symmetry axis than in figure $1 \mathrm{~d}$, so the latter could have a lower goodness than the former. On the other hand, it is also known that a central axis leads to a higher goodness than a peripheral one (Saarinen 1988; Locher and Nodine 1989; Herbert and Humphrey 1996), so that would cause an advantage for figure $1 \mathrm{~d}$ compared to figure $2 \mathrm{a}$. These factors can affect the processing of the symmetry but they had not been incorporated in the original HA (see discussion between Wagemans 1999 and van der Helm and Leeuwenberg 1999). 


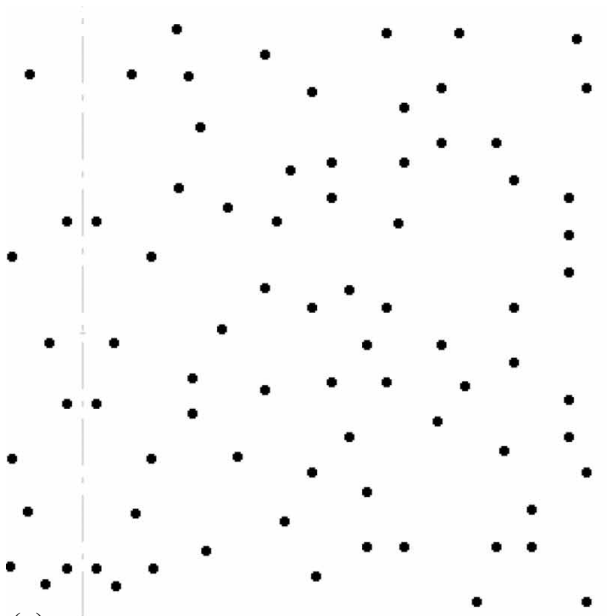

(a)

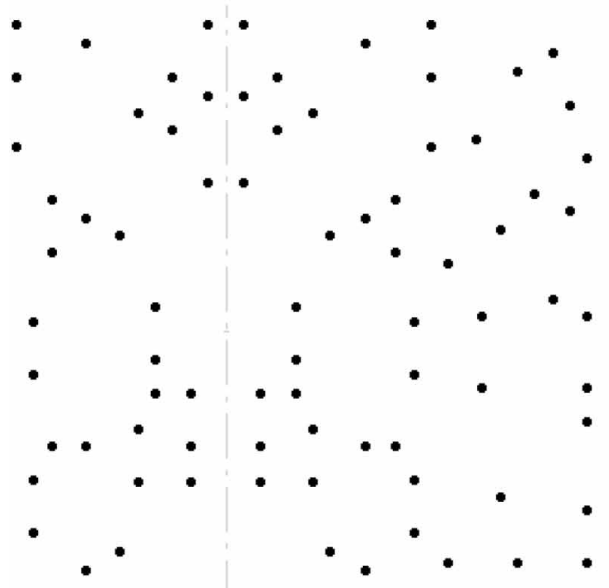

(c)

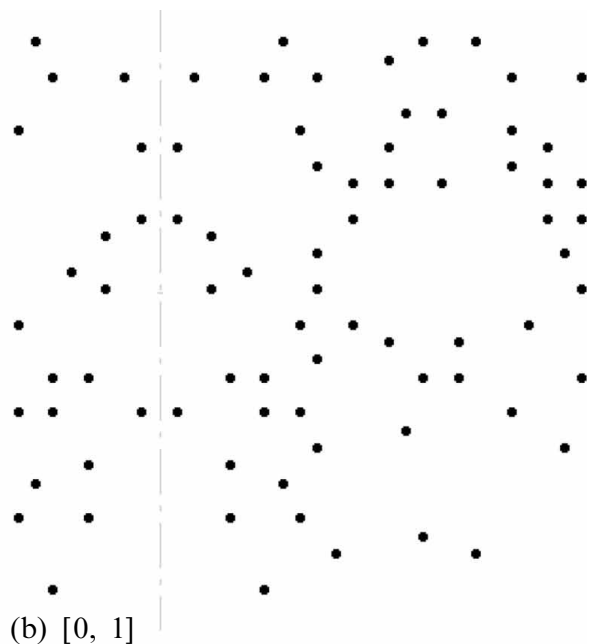

(b) $[0,1]$

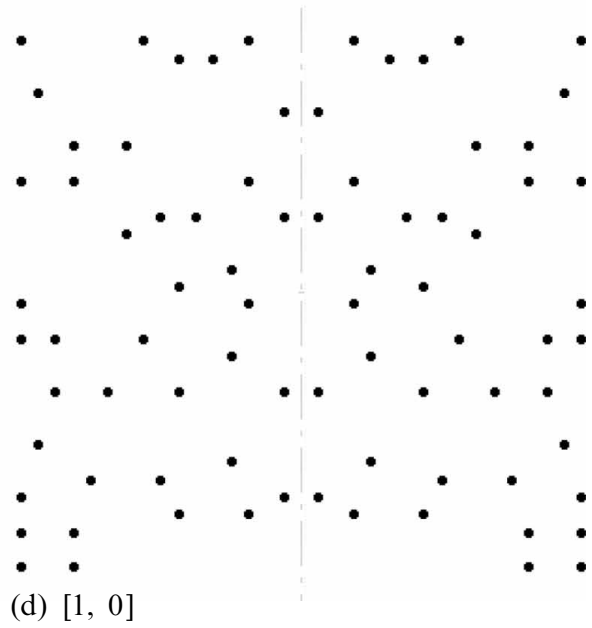

Figure 1. Different examples of symmetry. (a) Local symmetry which involves $25 \%$ of the dots. (b) Local symmetry which involves $50 \%$ of the dots. (c) Local symmetry which involves $75 \%$ of the dots. (d) Global symmetry which involves $100 \%$ of the dots. The code with brackets refers to the experimental conditions (see text).

It remains to be seen whether and how our empirical measure of goodness (see below) will distinguish these patterns. We believe that, contrary to the prediction based on the HA and the proximity to the axis, patterns with two local symmetries will have lower goodness than patterns with a simple global symmetry (compare figure $2 \mathrm{a}$ with figure $1 \mathrm{~d}$ ).

In a similar way, patterns with one global and two local axes of symmetry show the same amount of regularity as a double global symmetry, because each dot of these patterns is implied in two different symmetries (see figures $2 \mathrm{c}, 2 \mathrm{~d}$, and figure $3 \mathrm{a}$ ). HA predicts the same goodness also for those kinds of patterns, despite their different appearances. Figure 2c looks like two identical but distinct objects, whereas figure $3 \mathrm{a}$ is seen as one coherent configuration, although more complex than the former (probably based on the crossing axes). In a more general way, these examples show how the simultaneous presence of multiple symmetries creates the issue of possible interactions between the constituent regularities. More specifically, it can be supposed that two regularities can work in cooperation or in competition with one another, or, 


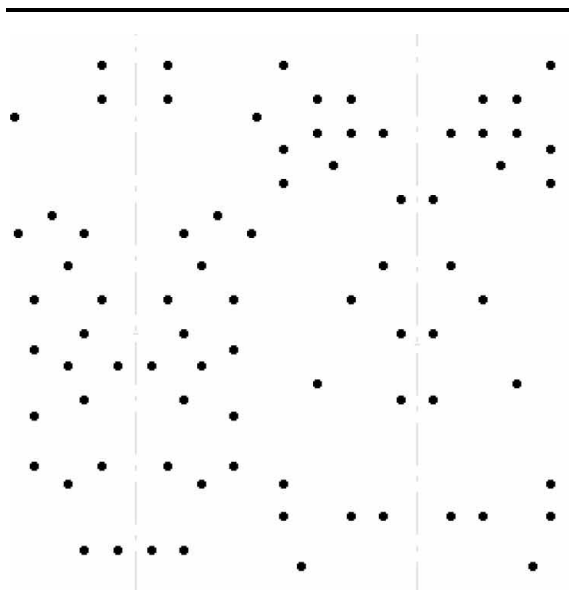

(a) $[0,2]$

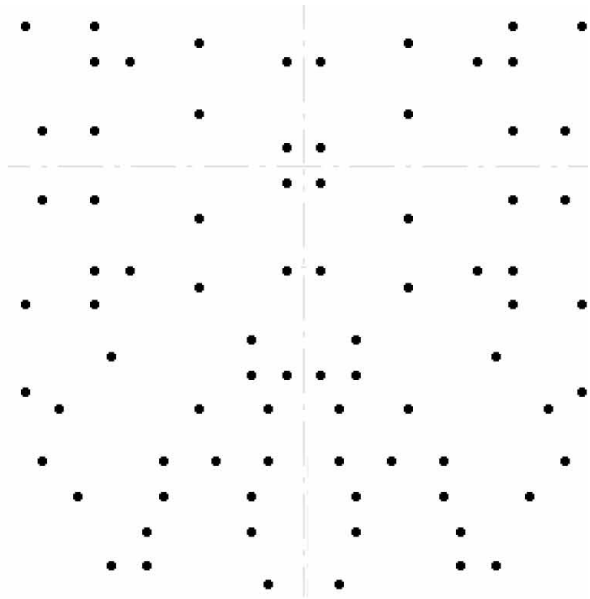

(b) $[1,1]$

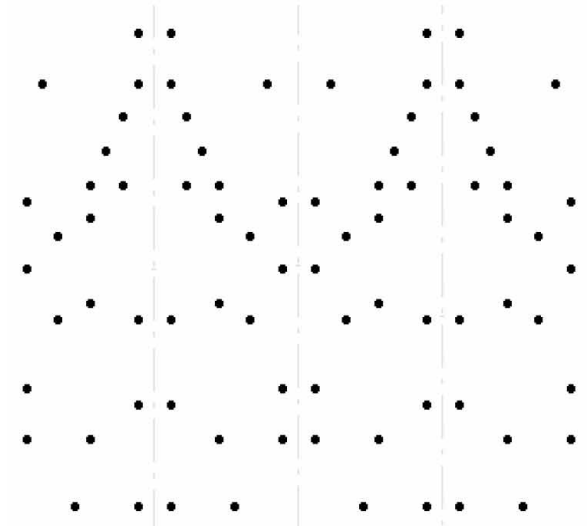

(c) $[1,2 \mathrm{a}]$

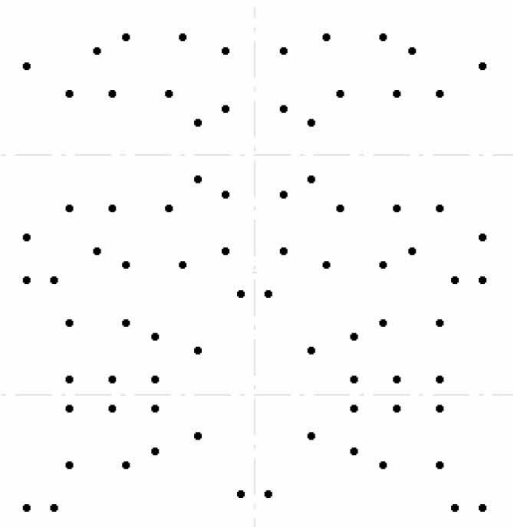

(d) $[1,2 b]$

Figure 2. Different examples of symmetry. (a) Two local symmetries, each involving $50 \%$ of the dots. (b) One global axis of symmetry with a single local axis of symmetry orthogonal to it. (c) One global axis of symmetry with two local axes of symmetry parallel to it. (d) One global axis of symmetry with two local axes of symmetry orthogonal to it. The code with brackets refers to the experimental conditions (see text).

finally, they can be indifferent to one another. When the latter is the case, it can be hypothesised that the goodness of the pattern is the simple addition of the goodness of the two regularities. In all other cases, their combination may not be simply additive.

\subsection{The present study}

In order to test this, we designed eight distinct kinds of configurations combining different numbers of local and global axes of symmetry (see table 1 for an overview).

It has been shown that an increase in the number of global symmetry axes indeed yields an increase in goodness, but not in a linear way (Palmer and Hemenway 1978; Wagemans et al 1991, 1993; Wenderoth and Welsh 1998; Friedenberg and Bertamini 2000). The HA also predicts a nonlinear increase of goodness for multiple global symmetry (for patterns with one to eight global symmetry axes, the $W$ values are 0.50 , $0.75,0.66,0.87,0.80,0.83,0.78,0.94$, respectively).

Consider the patterns (d) in figure 1, and (b) and (d) in figure 2. In the latter two patterns, single local symmetry and double local symmetry was added to a global symmetry pattern, respectively. For this kind of patterns, differently from the global axes 
Table 1. Representation of the stimulus conditions of the experiment. All symmetric stimuli were obtained by combining different numbers of local and global axes of symmetry. In this table broken line patterns instead of dots are used to increase the readability of the different kinds of configurations.

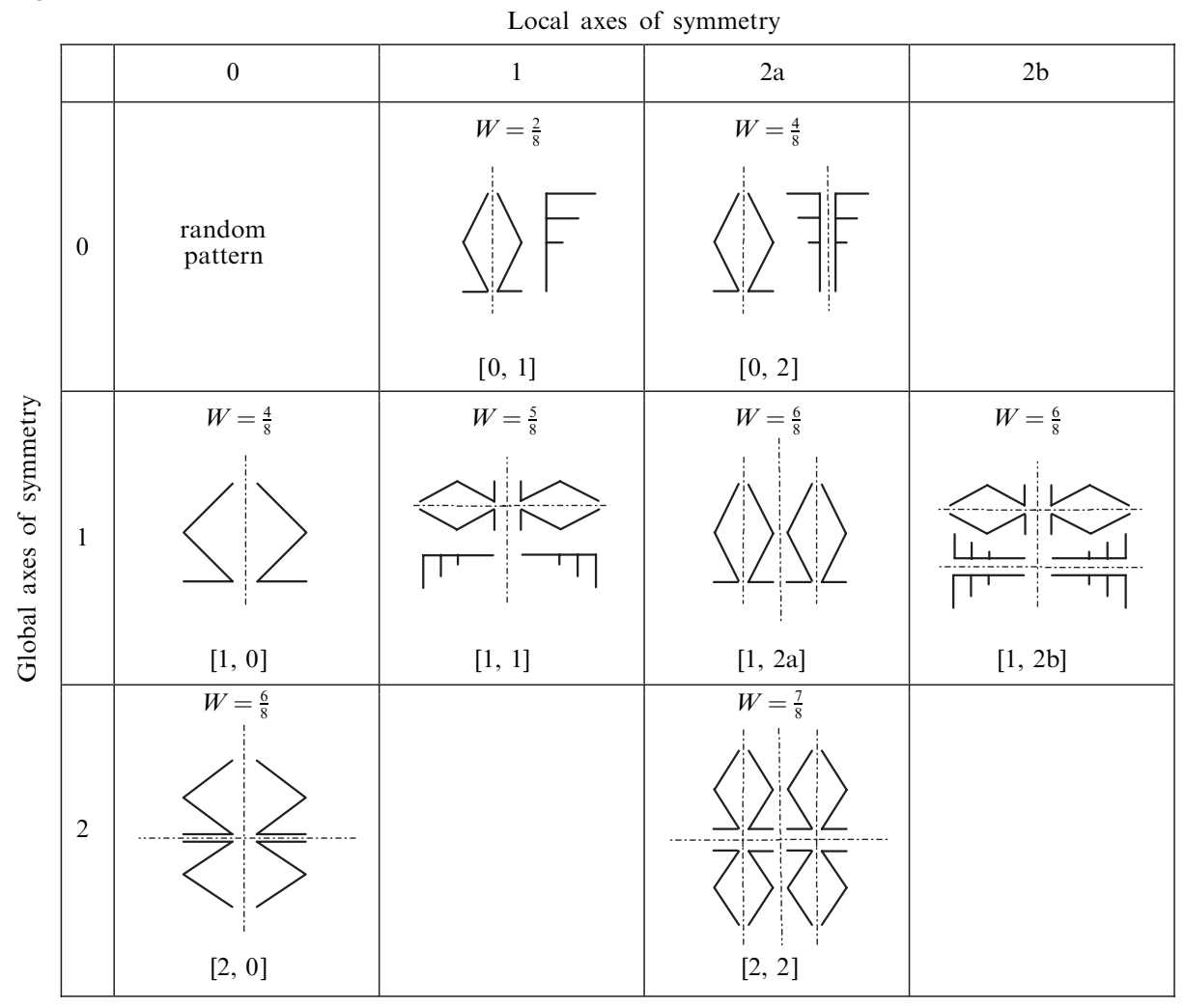

of symmetry, HA yields a monotonic increase of goodness, resulting from the addition of compatible symmetries. For patterns (d) in figure 1, and (b) and (d) in figure 2 the $W$ values are $0.5,0.625,0.75$, respectively (the latter two values resulting from the addition of 0.125 to 0.5 , one or two times, respectively). In contrast, from a phenomenological point of view, it is clear that they show different structures and appearances, resulting from interactions between the symmetries rather than from simply increasing the amount of regularities. As a consequence, we hypothesised the same nonlinear increase of goodness as observed for the increasing number of global symmetry axes.

In the two kinds of patterns labeled $2 \mathrm{c}$ and $2 \mathrm{~d}$, the orientation of the local axes was varied relative to the global ones (orthogonal versus parallel). The HA yields the same $W$ value for figures $2 \mathrm{c}, 2 \mathrm{~d}$, and also for figure $3 \mathrm{a}$ (pattern with double global symmetry). However, the pattern in figure $2 \mathrm{c}$ appears like a two sub-unit configuration and shows an ambiguous perceptual organisation resulting from a competition between substructures, whereas the pattern in figure $2 \mathrm{~d}$ does not. The latter pattern includes two crossings between the axes of symmetry, which seems to increase its complexity without affecting the stability of its perceptual organisation. For these reasons, we also predict a different overall goodness of these stimuli.

Finally, we propose the same prediction of nonlinearity in the combination of goodness values for a double global and double local symmetry (see figure 3b), for which the HA predicts the highest $W$ value between all patterns in table 1 .

Table 1 includes an overview of the eight different kinds of symmetry to be tested in the experiment below. All symmetries are a combination of 0,1 , and 2 global axes 


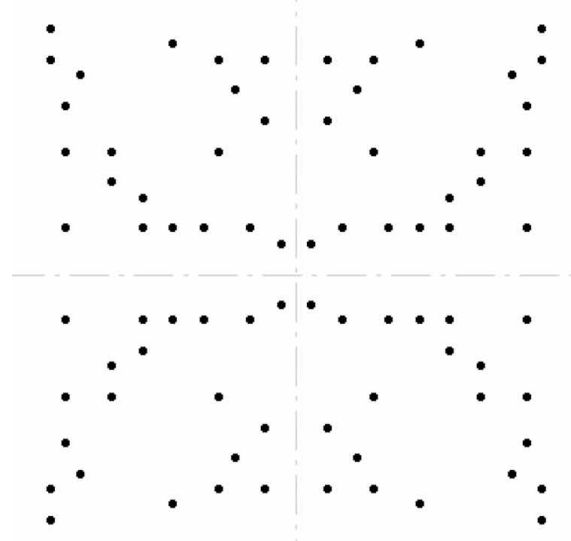

(a) $[2,0]$

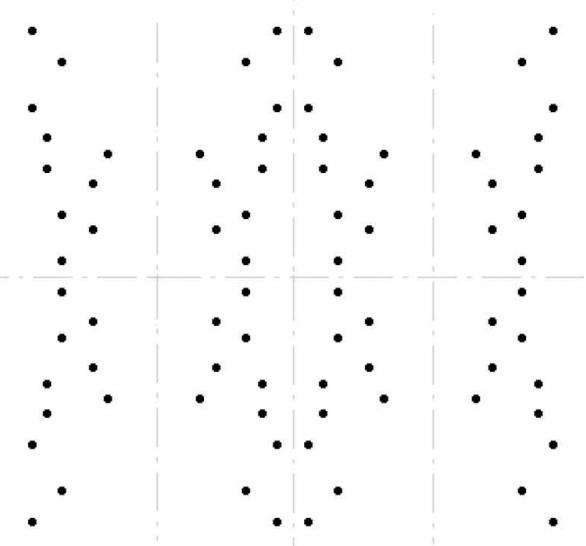

(b) $[2,2]$

Figure 3. Different examples of symmetry: (a) Two global axes of symmetry (orthogonal to one another). (b) Two global axes of symmetry (orthogonal to one another) and two parallel local axes of symmetry. The code with brackets refers to the experimental conditions (see text).

of symmetry (rows) with 0,1 , and 2 local axes of symmetry (columns). The line patterns shown in each entry of this table are icons used to illustrate the relations between the different components of a pattern. The code below each icon is the simple pairwise combination of the two variables (global and local symmetries). Above each icon is the pattern's calculated $W$ value, derived from the simplest possible HA model for compatible regularities (see above). When we find it relevant, we also distinguish between local axes being (a) parallel or (b) orthogonal to the global axis of symmetry. These codes are also shown in the stimulus examples (figures 1-3) and the results (figures 4-5 and table 2).

\subsection{Methodological considerations}

The operational definition of a pattern's goodness that we adopted assumes that there is a direct relationship between the overall goodness of a pattern and the detectability of any kind of regularity in the pattern. This is not necessarily the case, however, because other factors related to the experimental task or procedure may come into play as well. To illustrate this, it is worth mentioning that pilot experiments with a fixed presentation time for all conditions shown in table 1 had revealed a strong dissociation between two groups of conditions - those with no global symmetry on the one hand $([0,1]$ and $[0,2])$, and those with one or two global symmetries on the other hand $([1,0],[1,1],[1,2 \mathrm{a}],[1,2 \mathrm{~b}],[2,0],[2,2])$, with little or no difference between the conditions within each group. In other words, a single short presentation time would yield floor effects in the first group of difficult-to-detect symmetries and a single long presentation time would yield ceiling effects in the second group of easy-to-detect symmetries. Presenting each type of symmetry in a separate block of trials might appear a solution to this problem but it introduces another, equally disturbing factor. When presented with only one particular kind of symmetry in a homogeneous block of trials, subjects might adjust their viewing strategies and they might start looking for a particular localised component of the regularity rather than the global regularity whose overall goodness we want to measure. In other words, mixing the patterns together is the best way to avoid these strategic side-effects and to capture overall goodness in its purest perceptual form, but this induces a very large variability in the data and, consequently, a lack of power in the statistical analysis. To solve this conundrum we decided to use an adaptive procedure to obtain a balanced percentage of 
correct and incorrect responses in all eight kinds of symmetrical stimuli and to use the presentation time to obtain this response criterion as the major dependent variable.

Obviously, these issues could also be solved by changing the task. For instance, instead of asking for an overall regularity-detection task (any kind of regularity present or absent), it is also possible to ask the subject to discriminate local from global symmetries, or between the different numbers of symmetry axes, and so on. But, in this way, we obtain a measure of the discriminability between different kinds of symmetries and not a measure of the detectability of the regularities included in a pattern. In other words, the measure of goodness does not apply to the discrimination of different kinds of regularity but necessarily implies the detection of the overall regularity versus a random pattern. ${ }^{(2)}$

Another methodological issue concerns the position of the local axes of symmetry. In the experimental patterns, the number of dots implied in the symmetry co-varies with the spatial position of local symmetry axis (this is true also for the examples in figure 1). More precisely, all local symmetry axes do not pass through the centre of the pattern, whereas all global ones do (see table 1). It has been shown that spatial position in the visual field affects the goodness of a pattern (Saarinen 1988; Locher and Nodine 1989; Herbert and Humphrey 1996; see also Wagemans 1995). Consequently, a possible severe confounding arises between local versus global symmetry and the degree of proximity of the component symmetry to the fixation point. To use only symmetries with central axes might be a suitable solution in order to rule out the spatial position as a confounding variable, but then the number of possible combinations of local and global symmetries would decrease dramatically. To obtain the same salience of local and global axis of symmetry in different positions, we decided, instead, to present the fixation point in the centre of the screen and the patterns in one of four shifted random positions of the screen: up-right, up-left, down-right, downleft (see section 2.1 below). Because in our stimuli all the local symmetries are perfectly balanced in left-right positions (or up-down positions in the horizontal patterns) with respect to the centre of the patterns (which is also the position of the global symmetry axes, when they exist), the degree of proximity to the fixation point is absolutely balanced between local and global axes of symmetry. In other words, considering all the samples of each kind of pattern, the average distance from the fixation point for local and global axes of symmetry is identical.

\section{Experiment}

\subsection{Method}

2.1.1 Subjects. Thirteen subjects (six males) from the University of Leuven took part in the experiment. All subjects had normal or corrected-to-normal vision and were unaware of the purpose of the experiment.

2.1.2 Apparatus. Each stimulus was visible on a 13.3 inch screen with $60 \mathrm{~Hz}$ temporal resolution. Observers sat in a dark room and viewed the screen from about $80 \mathrm{~cm}$. Stimuli were presented with the E-Prime program.

2.1.3 Stimuli. All stimuli were black dot patterns on a white background, each consisting of 80 dots (each dot was a square of $5 \times 5$ squared pixels, without the 4 corner pixels).

(2) In some way, measuring interactions between regularities needs a sort of 'blending' between them. Any kind of regularity should be enough for subjects to conclude that there is regularity and we are then interested in how the presence of different regularities affects the detectability of the overall regularity. This is the reason why we have chosen this open definition of regularity, although using only symmetry as regularity, as we did in our experiment, turns the regularity-detection task into a symmetry-detection task, as a matter of fact. 
A computer algorithm was programmed in order to create them. The positions of the random dots were arranged within a square matrix of 1024 locations (32 rows $\times 32$ columns). The only constraint was that two dots could not be placed in two contiguous locations; that is, every dot had its eight adjacent locations free. The resulting pattern was included in a square area of $12 \mathrm{~cm} \times 12 \mathrm{~cm}$, so the pattern was about $8.6 \mathrm{deg}$ of visual angle wide. In total, 640 stimuli, all different from one another, were used: 320 were random and 320 were symmetrical. The latter were divided into 8 sets, each consisting of 40 different patterns, with the structure of the patterns as illustrated in table 1 and shown in figures $1 \mathrm{~b}, 1 \mathrm{~d}, 2$, and 3. Finally, all the symmetric patterns were presented in two orientations: 20 were presented horizontally and 20 vertically.

2.1.4 Experimental design. Table 1 illustrates the eight symmetry conditions obtained by the combination of different numbers of local and global axes of symmetry. Each of the two variables had three levels: $0,1,2$. However, the combination of two global and one local axis of symmetry is not feasible. The orientation of the local axes relative to the global one (orthogonal versus parallel) was varied only for stimuli with two local and one global axes of symmetry. In other words, the design was not a completely factorial design and could not be analysed as such (see below).

The task was a simple discrimination between random and symmetrical patterns. The notions of global and local symmetry were explained before the training and the experimental blocks of trials. Observers were shown all eight different kinds of possible patterns. Moreover, they were informed that $50 \%$ of the stimuli were random dot patterns and 50\% were symmetrical dot patterns (one of eight possible types). The stimuli were presented in a random order. The assignment of response key (left versus right) to response category (random versus symmetrical) was counterbalanced between subjects.

2.1.5 Procedure. Every trial consisted of the following sequence of events:

(i) A black fixation cross in the centre of the screen for $500 \mathrm{~ms}$.

(ii) A random or symmetrical pattern presented in one of four random positions of the screen: up-right, up-left, down-right, down-left. Each position was obtained by shifting the stimulus away from the centre of the screen by $2.7 \mathrm{~cm}$ two times: horizontally (either left or right) and vertically (either up or down). The presentation time of the pattern was variable between different blocks (see below).

(iii) A masking pattern in the centre of the screen for $1500 \mathrm{~ms}$, sufficiently large to cover the complete stimuli at all four possible positions. The mask pattern was randomly chosen from a set of ten different random-dot patterns consisting of 110 dots.

Immediately after the subject's answer, a feedback sound was presented. Correct and incorrect answers were followed by a $300 \mathrm{~ms}$ high-frequency tone $(1000 \mathrm{~Hz})$ and a $500 \mathrm{~ms}$ low-frequency tone $(400 \mathrm{~Hz})$, respectively.

Observers were given two training blocks, each consisting of 64 trials. The actual experiment consisted of five blocks of 128 trials each (64 random and 64 symmetrical). Observers could rest between every block.

The presentation time in the first and second training block was set at $400 \mathrm{~ms}$ and $200 \mathrm{~ms}$, respectively. As already mentioned (in section 1.4), during the experiment the presentation time was modified between the five different blocks.

So, in the first experimental block the presentation time was set at $150 \mathrm{~ms}$ for all patterns and in the following blocks the presentation time was variable depending on the number of correct and incorrect responses of the subjects. More precisely, at the beginning of every new block the presentation time for each pattern type was recalculated on the basis of the number of correct responses obtained on each pattern type in the previous block. The presentation time was left unchanged only when the correct responses constituted exactly $75 \%$ of the total responses (ie 6 out of 8 trials correct), 
and it was changed in every other case. It increased by $25 \mathrm{~ms}$ for every error when the number of correct responses was less than $75 \%$, and it decreased by $25 \mathrm{~ms}$ for every correct answer when the number of correct responses exceeded $75 \%$. The estimation of the criterion performance level of $75 \%$ was based on the combination of the two orientations (to have a more stable measure) but the adjustment procedure incorporated eventual differences between them. In order to make this adaptive procedure clearer, let us consider an example. Imagine that in one block, during the 8 presentations of the same pattern type (4 horizontal and 4 vertical), 3 errors are made: 2 for the horizontal and 1 for vertical patterns. Then, in the next block, the presentation time of the horizontal and the vertical patterns was increased by $50 \mathrm{~ms}$ and $25 \mathrm{~ms}$, respectively. Finally, because the random patterns were completely interspersed with the symmetrical ones, the presentation time for the random patterns was always set to the presentation time of the last-but-one trial.

The whole experiment, including instructions and practice blocks, took about $45 \mathrm{~min}$.

\subsection{Results}

As explained above, we preferred to use an adaptive procedure in which the presentation time for each type of symmetry decreases or increases between the five blocks in order to reach a performance level of $75 \%$ correct responses. In this way, the presentation time becomes a direct (although inverse) index of perceived goodness (shorter presentation time indicates higher goodness). At the beginning of the first block, the presentation time was equal for all stimuli $(150 \mathrm{~ms})$ and then it became gradually different for the different types of symmetry. On the basis of the number of correct responses obtained on each pattern type, the presentation time for each pattern type was recalculated for every new block. Although we ran only five blocks, using the accuracy of the last block it is possible to calculate the presentation time of a sixth 'virtual' block. Because after the fifth block the desired performance level (75\% of right answer) is not reached yet [see footnote (3)], the analysis was conducted on the average presentation time based on the fourth, fifth, and sixth ('virtual') blocks. This procedure reflects the actual performance reached at the end of the session better than using the presentation time of the third, fourth, and fifth blocks. The values obtained for horizontal and vertical patterns are shown in figures $4 \mathrm{a}$ and $4 \mathrm{~b}$, respectively. One subject was dropped from the analysis because he performed less than $60 \%$ correct for the random patterns. Overall, performance converged to $71 \%$ and $69 \%$ correct responses for symmetrical and random-dot patterns, respectively.
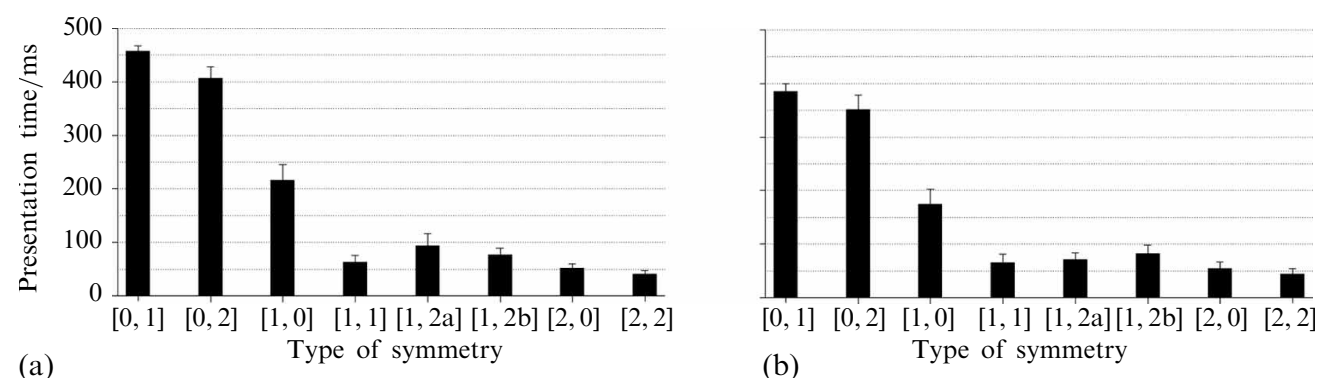

Figure 4. Results of the experiment. Average presentation time (of the last three experimental blocks) for the different types of symmetry: (a) horizontal patterns, (b) vertical patterns. The error bars represent the mean standard errors.

We computed the average presentation time separately for horizontal and vertical patterns, and then we performed, by means of paired samples $t$-tests, all possible paired comparisons. (We do not consider the main effects of both variables underlying our design, number of global and local axes of symmetry, because they were not combined 
factorially and because the predicted interactions required more specific tests anyway.) In order to determine whether the differences between the symmetry conditions were statistically significant, the false-discovery-rate (FDR) approach was used. This statistical procedure, introduced by Benjamini and Hochberg (1995), is effective in all those experimental contexts in which the computation of a large number of comparisons is required. FDR is the ratio between the number of errors committed when $\mathrm{H}_{0}$ is rejected $(V)$ and the total number of the rejected $\mathrm{H}_{0}(R)$. Benjamini and Hochberg's procedure ensures that the expected value of FDR will not be higher than $\alpha$ : formally $E(V / R) \leqslant \alpha$. Compared to the more traditional methods, the FDR procedure allows us to keep Type I error under control as well as increasing the statistical power. Unfortunately, the specific FDR calculation consists of several steps and it is rather complex to be explained briefly (for further explanation, see Benjamini and Hochberg 1995). ${ }^{(3)}$

Results show significant differences $(\alpha=0.01)$ between the two stimulus types with only one or two local axes of symmetry $([0,1]$ and $[0,2]$, respectively) and all the other stimuli (requiring longer presentation times). These two stimulus types differ significantly $(\alpha=0.01)$ from one another only in the horizontal condition, although there is a trend in the predicted direction also for the vertical condition. Stimuli with only one global axis of symmetry $([1,0])$ require significantly longer presentation times than all the others, while the remaining stimuli are not significantly different from one another, except for the double global and double local symmetry $([2,2])$ that differs significantly from both patterns with one global and two local symmetries $([1,2 \mathrm{a}]$ and $[1,2 \mathrm{~b}])$ in the horizontal condition and from one of them in the vertical conditions ([1,2b]) (see figure 4 and parts $\mathrm{a}$ and $\mathrm{b}$ of table 2). On combining horizontal and vertical conditions, significant differences arise from patterns with two global symmetries $([2,0])$ and both patterns with one global and two local symmetries $([1,2 \mathrm{a}]$ and $[1,2 \mathrm{~b}])$ (see part $\mathrm{c}$ of table 2$)$.

\subsection{Discussion}

The results clearly show that the detection of local symmetry is significantly more difficult when no global symmetry is involved. Presentation time average in patterns with one local symmetry is about double that found in patterns with one global symmetry (compare presentation time of conditions $[0,1]$ and $[1,0]$ in figure 4 , respectively). Hence, the goodness of symmetry decreases dramatically when the regularity does not affect the figure as a whole. The $W$ value calculated by HA for patterns with a single local symmetry is exactly half of the $W$ value for patterns with a single global symmetry. This prediction is matched almost exactly by our results. However, the HA predicts the same value of goodness for patterns with one global axis of symmetry and for patterns with two local axes of symmetry, because they have the same number of dots involved in the symmetry. This prediction is disconfirmed by our experiment: our results show a significant difference between conditions $[1,0]$ and $[0,2]$, with the former requiring almost half the time of the latter.

(3) The results reported in figure 4 probably underestimate the differences between the conditions. There are reasons to believe that the adaptive procedure has not actually converged to the desired performance level after only five blocks. The average performance levels for the last three blocks in the different conditions (in the order of figure 4) are $0.24,0.37,0.57,0.82,0.76,0.87,0.83$, and 0.89 , for the horizontal patterns, and $0.37,0.48,0.73,0.83,0.84,0.81,0.83$, and 0.91 , for the vertical patterns, respectively. However, these performance levels suggest an even stronger effect on goodness rather than a speed-accuracy tradeoff. Taking only the means of the final block would yield larger numerical differences between the experimental conditions but the statistical power would be less. Likewise, running more blocks could have resulted in a more powerful design but after 45 min, we noticed increasing levels of fatigue and some lack of motivation on our subjects' part. However, for the main point of the present paper, the confirmation that goodness depends on interactions between symmetries, the current procedure and design were clearly sufficient. 
Table 2. $t$-Tests and FDR-adjusted $p$ values for all the possible paired comparisons of stimulus conditions for (a) horizontal, (b) vertical, and (c) combined patterns. Inside each cell the first number indicates the $t$-test value and the second one the FDR-adjusted $p$ value. The symbols (*) and $(* *)$ indicate whether the test is statistically significant at $5 \%$ or $1 \%$ level, respectively.
$[0,1]$
$[0,2]$
$[1,0]$
$[1,1]$
$[1,2 \mathrm{a}]$
$[1,2 b]$
$[2,0]$

(a) Horizontal patterns

$[0,2] \quad 2.73$

$0.026^{*}$

$[1,0] \quad 5.95 \quad 5.13$

$0.000 * * \quad 0.000 * *$

$[1,1]$

$6.92 \quad 6.65$

$\begin{array}{ll}{[1,2 \mathrm{a}]} & 6.66 \\ & 0.000 * *\end{array}$

$0.000 * *$

$[1,2 \mathrm{~b}]$

$0.000 * *$

$0.000 * *$

6.31
$0.000 *$

6.91
$0.000 * *$

6.62

$[2,0]$

6.97
$0.000 * *$

$[2,2]$

6.99
$0.000 * *$

$0.000 * *$

6.72

$0.000 * *$

6.76

$0.000 * *$

(b) Vertical patterns

$\begin{array}{ll}{[0,2]} & 1.50 \\ & 0.213\end{array}$

$[1,0]$

$[1,1]$

5.63

$0.000 * *$

4.72

$0.001 * *$

$[1,1]$

6.67

$0.000 * *$

6.16

$0.000 * *$

3.94

$[1,2 \mathrm{a}]$

6.23

\subsection{3}

$0.000 * *$

$0.000 * *$

6.09

$[1,2 \mathrm{~b}]$

6.65

$0.000^{* *}$

$0.000 * *$

$[2,0]$

$[2,2]$

6.78
$0.000 * *$

6.31

$0.000 * *$

6.39

$0.000 * *$

$0.000 * *$

$0.003 * *$

$3.96 \quad 0.43$

$0.003 * * \quad 0.672$

$3.51 \quad 1.09$

$0.007 * *$

0.347

$4.36 \quad 0.69$

$0.002 * * \quad 0.523$

$4.67 \quad 1.47$

$0.001 * * \quad 0.213$

(c) Combined horizontal and vertical patterns

$[0,2] \quad 2.67$

0.018 *

$[1,0]$

8.10

6.92

$[1,1$

$0.000 * *$

$[1,1]$

9.64

$0.000 * *$

$[1,2 \mathrm{a}]$

9.47

$0.000 * *$

[1, 2b]

9.62

0.000 **

$[2,0]$

9.76

$[2,2]$

0.000 **

9.81

$0.000 * *$

$0.000 * *$

\subsection{3}

0.000 **

\subsection{9}

0.000 **

9.06

0.000 **

9.28

0.000 **

9.36

$0.000 * *$

6.33

0.000 **

5.52

0.000 **

5.86

0.000 **

6.83

0.000 **

7.17

$0.000 * *$

1.48

0.162

1.52

0.158

1.22

0.241

2.49

$0.024 *$
0.82

0.464

$1.33 \quad 1.92$

$0.255 \quad 0.111$

$2.33 \quad 2.74$

0.056
1.30

0.242

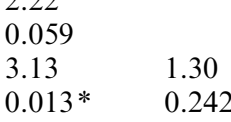

Let us now compare condition $[1,1]$ with condition $[1,2 \mathrm{a}]$ or $[1,2 \mathrm{~b}]$ (see tables 1 and 2), where the latter two patterns include one local symmetry more than the former. No significant difference between these configurations was found, although HA yields lower $W$ values for condition $[1,1]$ than for both [1,2a] and [1,2b] (see tables 1 and 2). This deviation between results and prediction cannot be due to a statistical power 
problem because the trends clearly go in the opposite direction: the $[1,2 \mathrm{x}]$ conditions tend to require more time than $[1,1]$, not less, as expected from their higher $W$ values. Another mismatch with HA's prediction is obtained with conditions [2,0] and [2, 2] (see tables 1 and 2), even though the second one includes two more local symmetries than the first one. In this case, we must admit the possibility of a floor effect masking any true difference, because here the presentation times were very short already.

Data analysis also reveals no significant difference between conditions [1, 2a] and $[1,2 \mathrm{~b}]$, despite their very different appearance (see figures $2 \mathrm{c}$ and $2 \mathrm{~d}$ ). Stimulus [1, 2a] appears as a configuration consisting of two sub-units, while stimulus $[1,2 b]$ is seen as a single coherent configuration with two crossings between the axes of symmetry. Perhaps the lack of a difference can be explained as follows. Although pattern 2c consists of two objects, which should reduce the goodness, both objects are identical and probably the repetition pops out as a new regularity, which may increase the overall goodness again. So, the two local symmetries in $[0,2]$ do not add up to one global symmetry (as in $[1,0]$ ); hence, they remain separate and thus they tend to compete, while the two local symmetries in [1,2a] are either seen as one global symmetry with some extra local regularity (as in $[1,1]$ or $[1,2 \mathrm{~b}]$ ) or as two identical objects, each with a global symmetry of half that size.

In order to have a stronger comparison between the theoretical value of goodness calculated by HA and the results we obtained, it is possible to use a simple linear model. Plotting the $W$ value and the presentation time of each type of patterns and calculating a regression between these two sets of values, it is then possible to consider the distance between each point and the regression line as a value of the fit of HA to our results (see figure 5). Patterns [0,2], [1, 1], and [2,2] are the ones with the longer distance from the regression line, both in the horizontal and the vertical condition. ${ }^{(4)}$ From this further analysis, it is possible to affirm that HA overestimates the goodness of pattern $[0,2]$ and underestimates the goodness of $[1,1]$. The first of these results can be due to the role of symmetry in segregating objects from the background. As already mentioned, condition $[0,2]$ shows a pattern in which two sub-units pop out as distinct objects and this may produce an increasing difficulty in regularity detection (a two-object cost is a well-known effect in several domains - eg Baylis 1994; Hulleman

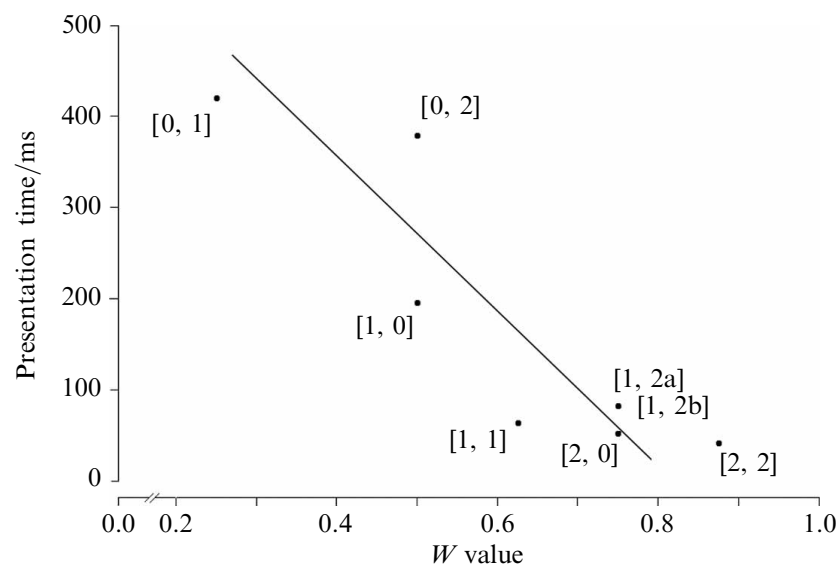

Figure 5. Average presentation time of combined data from horizontal and vertical condition plotted with their corresponding $W$ value. The segment is the regression line between them.

(4) In figure 5 (combined data from horizontal and vertical condition) residual values are the horizontal distance between each $W$ value and the regression line. In this condition the residual values are (in the order of figure 4) $-0.074,0.126,-0.088,-0.118,0.028,0.025,-0.005,0.106$, respectively (in the horizontal and vertical condition residual values are almost the same). 
and Boselie 1997; Friedenberg and Bertamini 2000; Bertamini et al 2002; Koning and Wagemans 2008). We label this the 'objectness issue'. The underestimation of the goodness of $[1,1]$ is more difficult to explain. A possible hypothesis is that the crossing between the local and the global axes of symmetry is highly salient (much like a local double symmetry), so that pattern $[1,1]$ has a goodness quite similar to pattern $[2,0]$. Anyway, this case seems to suggest a cooperation between regularities - that is when the overall detectability of two regularities is more than the sum of their single detectabilities. Our data also show that the goodness of pattern $[2,2]$ is overestimated by HA but, as suggested before, this observation should not receive too much weight because a possible floor effect may be at stake here.

In a more general way, the regression between the average presentation time of patterns and their corresponding $W$ value shows a rather good prediction of goodness by the HA $\left(R^{2}=0.80\right)$, although the results presented above may inspire some further fine-tuning of it, specifically with respect to the underestimation of the goodness of $[1,1]$. With respect to the overestimation of the goodness of $[0,2]$, it must be said that $\mathrm{HA}$ is a theory about the goodness of single and hierarchically nested regularities and then patterns $[0,2]$ are outside its primary scope (without this kind of patterns the regression gives a much better fit: $R^{2}=0.88$ ). Nevertheless, this result could be useful to inspire a future expansion of HA's scope.

In conclusion, the general hypothesis by which performance in regularity detection does not increase in a linear way, whenever an extra regularity is added, was confirmed. This result supports the role of interactions between regularities in determining the overall pattern goodness, as implied by the original Gestalt notion of goodness. Combining local symmetries seems to be a suitable and promising method for a deeper understanding of pattern goodness.

Acknowledgments. This research was supported by a research grant from the Fund for Scientific Research (FWO-Vlaanderen G.0095.03) and from the University Research Council (GOA/05/003) to JW. We wish to thank Perter van der Helm, Peter Wenderoth, and two anonymous reviewers for their helpful comments on a previous version of this paper.

\section{References}

Baylis G C, 1994 "Visual attention and objects: Two-object cost with equal convexity" Journal of Experimental Psychology: Human Perception and Performance 20 208-212

Baylis G C, Driver J, 1995 "Obligatory edge assignment in vision: The role of figure and part segmentation in symmetry detection" Journal of Experimental Psychology: Human Perception and Performance $211323-1342$

Benjamini Y, Hochberg Y, 1995 "Controlling the False Discovery Rate: a practical and powerful approach to multiple testing" Journal of the Royal Statistical Society B 57289 -300

Bertamini M, Friedenberg J, Argyle L, 2002 "No within-object advantage for detection of rotation" Acta Psychologica 111 59-81

Bertamini M, Friedenberg J D, Kubovy M, 1997 "Detection of symmetry and perceptual organization: The way a lock-and-key process works" Acta Psychologica 95119 - 140

Bruce V, Morgan M J, 1975 "Violations of symmetry and repetition in visual patterns" Perception $4239-249$

Corballis M C, Roldan C E, 1974 "On the perception of symmetrical and repeated patterns" Perception \& Psychophysics $16136-142$

Csatho A, Vloed G van der, Helm P van der, 2003 "Blobs strengthen repetition but weaken symmetry" Vision Research 43993 - 1007

Csatho A, Vloed G van der, Helm P van der, 2004 "The force of symmetry revisited: Symmetryto-noise ratios regulate (a)symmetry effects" Acta Psychologica 117 233-250

Dastani M, Scha R, 2003 "Languages for Gestalts of line patterns" Journal of Mathematical Psychology 47 429-449

Field D J, Hayes A, Hess R F, 2000 "The roles of polarity and symmetry in the perceptual grouping of contour fragments" Spatial Vision $1351-66$ 
Foster D H, Kahn J I, 1985 "Internal representations and operations in the visual comparison of transformed patterns: Effects of pattern point-inversion, positional symmetry, and separation” Biological Cybernetics $51305-312$

Friedenberg J, Bertamini M, 2000 "Contour symmetry detection: The influence of axis orientation and number of objects" Acta Psychologica $105107-118$

Helm P A van der, Leeuwenberg E L J, 1991 "Accessibility: A criterion for regularity and hierarchy in visual pattern codes" Journal of Mathematical Psychology 35 151-213

Helm P A van der, Leeuwenberg E L J, 1996 "Goodness of visual regularities: A nontransformational approach" Psychological Review 103 429-456

Helm P A van der, Leeuwenberg E L J, 1999 "A better approach to goodness: Reply to Wagemans (1999)" Psychological Review $106629-656$

Helm P A van der, Leeuwenberg E L J, 2004 "Holographic goodness is not that bad: Reply to Olivers, Chater, and Watson (2004)" Psychological Review 111261 - 273

Herbert A M, Humphrey G K, 1996 "Bilateral symmetry detection: testing a 'callosal' hypothesis" Perception $25463-480$

Hulleman J, Boselie F, 1997 "Visual attention and objects: New tests of two-object cost" Psychonomic Bulletin \& Review $4367-373$

Julesz B, 1971 Foundations of Cyclopean Perception (Chicago, IL: University of Chicago Press)

Koffka K, 1935 Principles of Gestalt Psychology (New York: Harcourt-Brace)

Koning A, Wagemans J, 2008 "Detection of symmetry and repetition in one and two objects: Structure versus strategies" Experimental Psychology forthcoming

Leeuwenberg E L J, 1969 "Quantitative specification of information in sequential patterns" Psychological Review 76 216-220

Leeuwenberg E L J, 1971 "A perceptual coding language for visual and auditory patterns" American Journal of Psychology 84 307-349

Locher P, Nodine C, 1989 "The perceptual value of symmetry" Computers \& Mathematics with Applications $17475-484$

Locher P, Wagemans J, 1993 "The effects of element type and spatial grouping on symmetry detection" Perception $22565-587$

Mach E, 1886 Beiträge zur Analyse der Empfindungen (Jena: Gustav Fisher) [republished as a translation from the 5th German edition revised and supplemented by S Waterlow The Analysis of Sensations and the Relation of the Physical to the Psychical (1959, New York: Dover)]

Olivers C N L, Chater N, Watson G D, 2004 "Holography does not account for goodness: A critique of van der Helm and Leeuwenberg (1996)" Psychological Review $111242-260$

Palmer S E, 1982 "Symmetry, transformation, and the structure of perceptual systems", in Organization and Representation in Perception Ed. J Beck (Hillsdale, NJ: Lawrence Erlbaum Associates) pp $95-144$

Palmer S E, 1983 "The psychology of perceptual organization: A transformational approach", in Human and Machine Vision Eds J Beck, B Hope, A Rosenfeld (New York: Academic Press) volume 1 , pp $269-339$

Palmer S E, Hemenway K, 1978 "Orientation and symmetry: Effects of multiple, rotational, and near symmetries" Journal of Experimental Psychology: Human Perception and Performance 4691 - 702

Royer F L, 1981 "Detection of symmetry" Journal of Experimental Psychology: Human Perception and Performance $71186-1210$

Saarinen J, 1988 "Detection of mirror symmetry in random-dot patterns at different eccentricities" Vision Research $28755-759$

Wagemans J, 1995 "Detection of visual symmetries" Spatial Vision 9 9-32

Wagemans J, 1999 "Toward a better approach to goodness: Comments on Van der Helm and Leeuwenberg (1996)" Psychological Review 106 610-621

Wagemans J, Gool L van, D’Ydewalle G, 1991 "Detection of symmetry in tachistoscopically presented dot patterns: Effects of multiple axes and skewing" Perception \& Psychophysics 50 $413-427$

Wagemans J, Gool L van, Swinnen V, Horebeek J van, 1993 "Higher-order structure in regularity detection" Vision Research 331067 - 1088

Wenderoth P, 1995 "The role of pattern outline in bilateral symmetry detection with briefly flashed dot patterns" Spatial Vision $957-77$

Wenderoth P, Welsh S, 1998 "Effects of pattern orientation and number of symmetry axes on the detection of mirror symmetry in dot and solid patterns" Perception $27965-976$ 


\section{PERTEPTION}

VOLUME 362007

www.perceptionweb.com

Conditions of use. This article may be downloaded from the Perception website for personal research by members of subscribing organisations. Authors are entitled to distribute their own article (in printed form or by e-mail) to up to 50 people. This PDF may not be placed on any website (or other online distribution system) without permission of the publisher. 